\title{
Medial Elbow Mass in an 18-year-old Woman
}

\author{
Kandice R. Beenken DO, Brian E. Walczak DO
}

Received: 28 September 2014/ Accepted: 28 January 2015/Published online: 11 February 2015

(C) The Association of Bone and Joint Surgeons (R 2015

\section{History and Physical Examination}

An 18-year-old right-hand dominant woman presented with an enlarging right medial elbow mass which was associated with worsening paresthesias during the past 2 months, primarily affecting her ring and small fingers. She denied any weakness or loss of function. There was no family history of soft tissue tumors, neurocutaneous disorders, or neuropathy. Physical examination revealed an elongated soft tissue mass along the posteromedial aspect of her right elbow. The mass was fairly firm, did not transilluminate, and was mobile in the mediolateral plane. Although the mass was not tender to palpation, percussion of the mass reproduced her paresthesias (a positive Tinel's sign). Moreover, light touch sensation was intact. Further evaluation of her right upper extremity revealed normal ROM, vascular status, and no atrophy of the upperextremity muscles. Small finger abduction strength was $4 / 5$

Each author certifies that he or she, or a member of his of her immediate family, has no commercial associations (eg, consultancies, stock ownership, equity interest, patent/licensing arrangements, etc) that might pose a conflict of interest in connection with the submitted article.

All ICMJE Conflict of Interest Forms for authors and Clinical Orthopaedics and Related Research editors and board members are on file with the publication and can be viewed on request.

Each author certifies that his or her institution approved or waived approval for the reporting of this case and that all investigations were conducted in conformity with ethical principles of research.

\section{K. R. Beenken ( $\square)$}

McLaren Macomb, 1000 Harrington Blvd, Mount Clemens,

MI 48043, USA

e-mail: kandice.beenken@mclaren.org

B. E. Walczak

McLaren Cancer Institute, Ted B. Wahby Cancer Center, Mount

Clemens, MI, USA with remaining strength testing revealing no other weakness. There was no associated lymphadenopathy.

Laboratory tests, including a complete blood count with differential, a comprehensive metabolic panel, sedimentation rate, C-reactive protein, and lactate dehydrogenase levels, were normal.

Imaging studies included AP and lateral radiographs of the humerus (Fig. 1) and MRI (Fig. 2).

Based on the history, physical examination, laboratory studies, and imaging studies, what is the differential diagnosis at this point?

\section{Imaging Interpretation}

The AP and lateral radiographs of her right humerus showed no observable soft tissue mass, calcification, or osseous abnormalities (Fig. 1).

The MR images showed an elongated lesion with a fusiform pattern of enlargement that traveled along the course of the ulnar nerve in the cubital tunnel and measured $10.2 \mathrm{~cm}$ craniocaudal $\times 1.5 \mathrm{~cm}$ transverse. The lesion was indistinguishable from the nerve and isointense with muscle on T1-weighted MR images (Fig. 2A, B), hyperintense to muscle on proton density MR images (Fig. 2C, D), and showed peripheral enhancement on T1-weighted fat suppressed postgadolinium MR images (Fig. 2E, F).

\section{Differential Diagnosis}

Benign peripheral nerve sheath tumors

Chronic inflammatory demyelinating polyneuropathy

Intraneural perineurioma

Lymphoma

Malignant peripheral nerve sheath tumors 


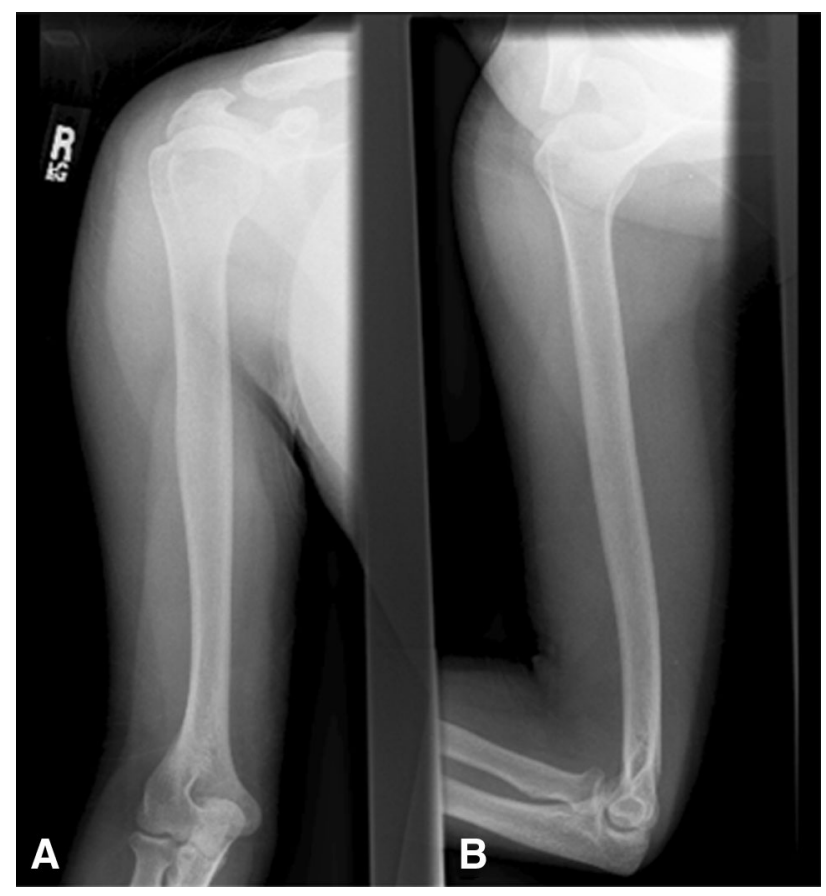

Fig. 1A-B (A) AP and (B) lateral radiographs of the patient's right humerus show no observable soft tissue mass, calcifications, or osseous abnormalities.

A CT-guided core needle biopsy of the lesion was performed and histologic features of the lesion were examined (Fig. 3).

Based on the history, physical examination, laboratory studies, imaging studies, and histologic picture, what is the diagnosis and how should the patient be treated?

\section{Histology Interpretation}

Microscopic examination (Fig. 3) showed plump perineurial cells with a "pseudo-onion bulb" appearance around a central nerve axon and Schwann cells. Epithelial membrane antigen immunostaining (Fig. 4) was positive in the perineurial cells and negative in the central Schwann cells. S100 stain (Fig. 5) showed a positive central stain in the Schwann cells and negative in the surrounding perineurial cells. CD34 and CD31 stains were negative in the lesion.

\section{Diagnosis}

Intraneural perineurioma.

\section{Discussion and Treatment}

Intraneural perineurioma is a rare entity affecting young adults, most prevalently during the second decade of life,
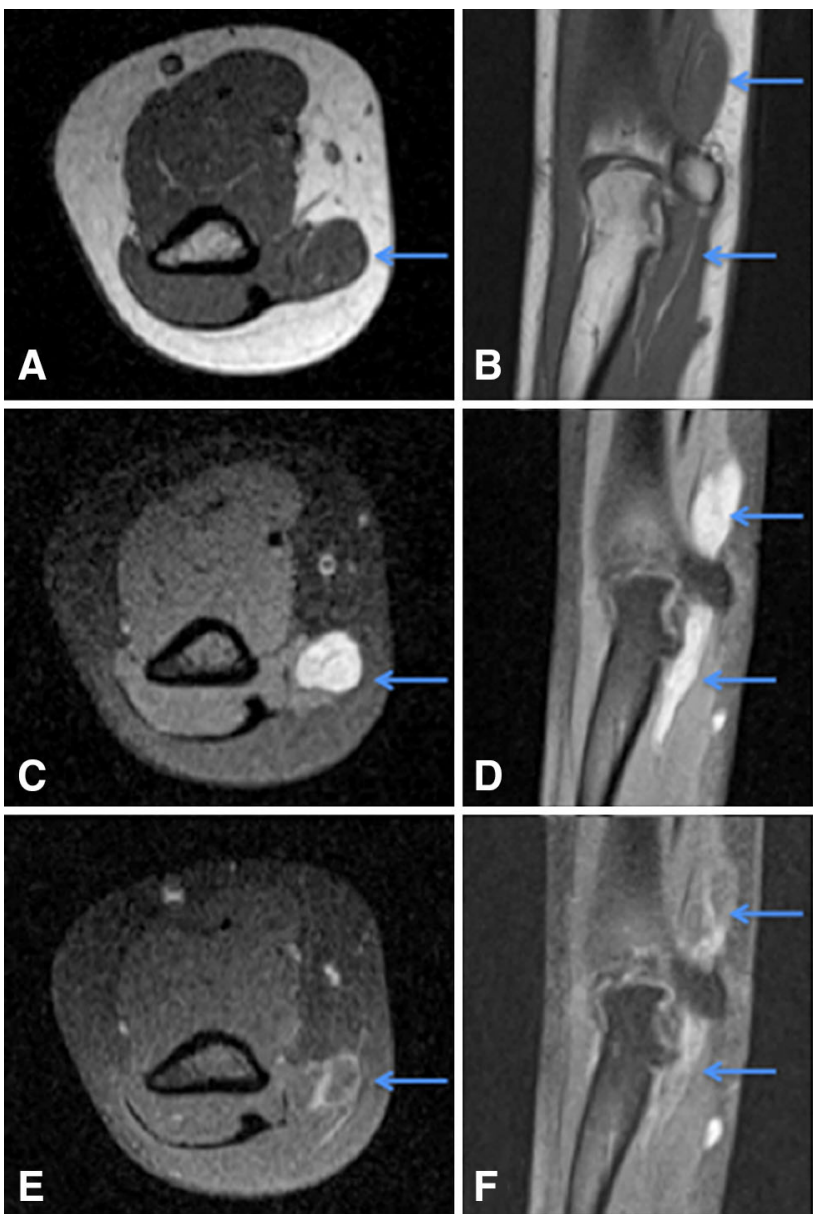

Fig. 2A-F (A) Axial T1-weighted turbo spin echo; (B) coronal T1weighted turbo spin echo; (C) axial proton density fat-saturated; (D) coronal proton density fat-saturated; (E) axial T1-weighted fat suppressed postgadolinium; and (F) coronal T1-weighted fat suppressed postgadolinium sequences show an elongated lesion, indistinguishable from the nerve, with a fusiform pattern of enlargement that travels along the course of the ulnar nerve in the cubital tunnel and is isointense to muscle on T1 sequences, hyperintense to surrounding muscle on proton density sequences, and peripheral enhancement on postgadolinium sequences.

and affects males and females equally. [6, 12, 13]. In one study, a predilection for the upper extremity was reported [6]; however, research has found a nearly equal incidence of upper and lower extremity involvement [12-14]. The definitive cause of these tumors is still being investigated $[5,10]$.

Patients typically present with a painless mononeuropathy, which includes progressive weakness throughout affected muscles, classically sparing sensation $[6,11,16]$. In the largest study to date, 29 patients presented with a chief complaint of weakness or atrophy and only three with numbness or pain. All 32 patients eventually had weakness develop during the study period. The distribution of neuropathy was purely motor in five patients, predominantly motor in 26 patients, and motor and sensory in one patient 


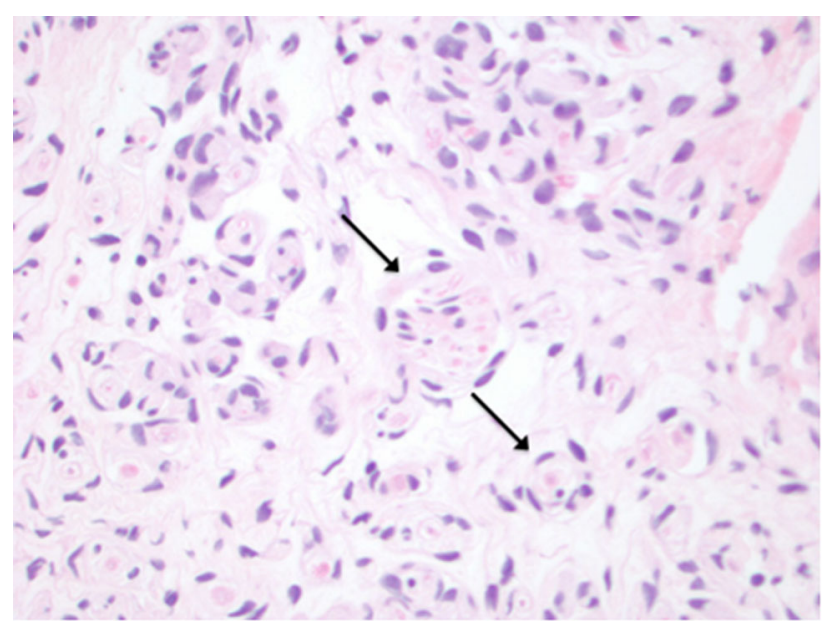

Fig. 3 Microscopic examination shows a "pseudo-onion-bulb" pattern composed of concentric layers of perineurial cells ensheathing a central axon and Schwann cells at the arrows (Stain, hematoxylin \& eosin; original magnification, $\times 200$ ).

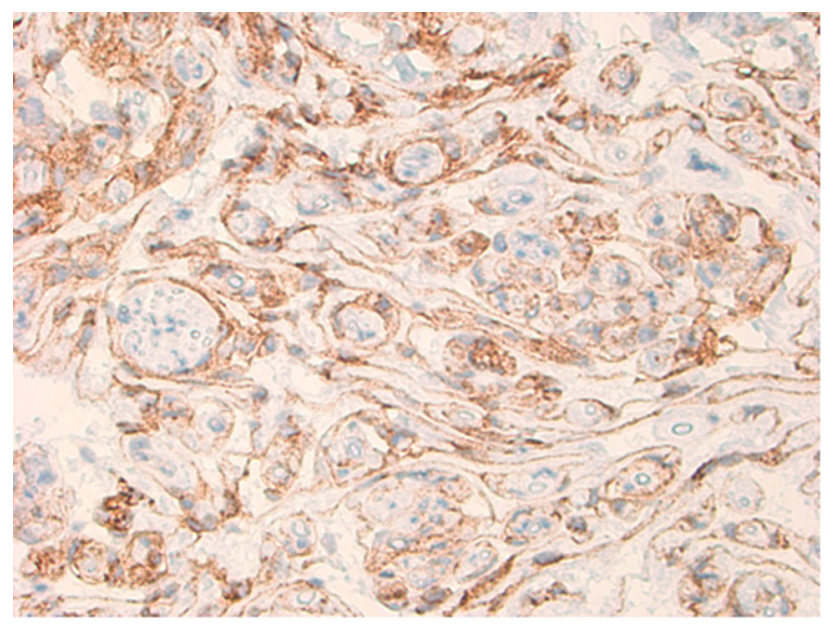

Fig. 4 Immunostain for epithelial membrane antigen highlights the perineurial cells (Original magnification, $\times 400$ ).

[13]. Our patient presented with the chief complaint of paresthesias and had 4/5 weakness with small finger abduction on physical examination.

The most sensitive imaging modality is MRI, on which an intraneural perineurioma is observed as a segment of fusiform enlargement with proximal and distal tapering [1, 13, 14]. The affected segment is hypointense to isointense to muscle on T1-weighted images and hyperintense to muscle on T2-weighted images. Most often there is avid, homogeneous, gadolinium enhancement [1, 13, 14]; however, minimal to no postcontrast enhancement has been reported [4, 17]. Mauermann et al. [13] found nerve enlargement ( 29 of 32), usually fusiform (27 of 32), isointense to muscle on T1-weighted images (27 of 32), hyperintense to muscle on T2-weighted images (25 of 32 ), and avid

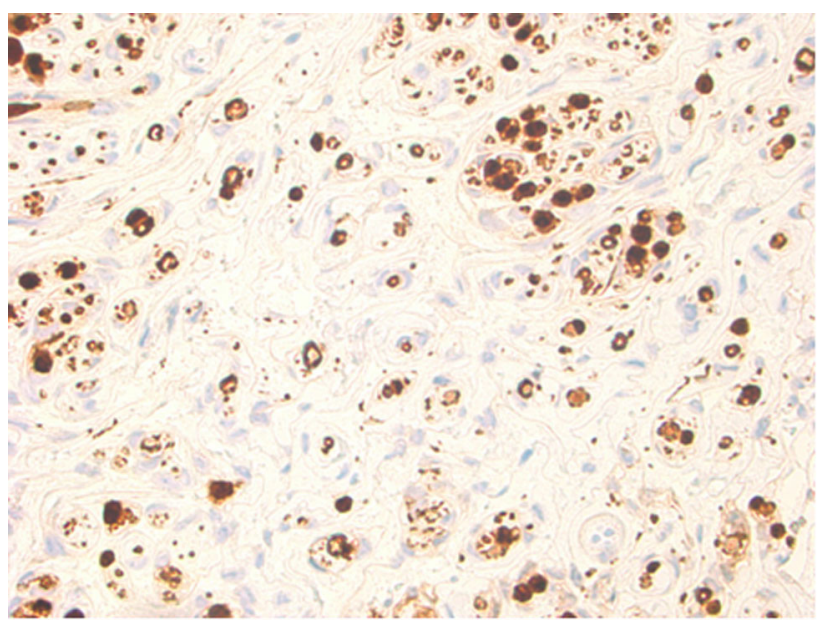

Fig. 5 Immunostain for S100 shows a reverse staining pattern, highlighting the central axon and Schwann cells, and not staining the perineurial cells (Original magnification, $\times 400$ ).

contrast enhancement ( 20 of 20 ) in all patients who received intravenous gadolinium. In our patient, the MRI appearance of the lesion was unique, as the lesion showed typical T1and T2-weighted imaging with mainly peripheral enhancement rather than central solid enhancement.

The definitive diagnosis of intraneural perineurioma was made by the characteristic pathology findings of concentric whorls of perineurial cells or "pseudo-onion bulbs," that stain positive for epithelial membrane antigen, highlighting the perineurial cells $[2,14]$. Additionally, staining negative for $\mathrm{S} 100$ protein differentiated perineurial cells from more common "onion-bulb" nerve sheath tumors (eg, schwannomas) [2, 14]. The concentric layers of cells seen resemble Schwann cell neoplasms which arise from the endoneurial layer surrounding the individual nerve axons as opposed to an intraneural perineurioma that arises from the perineurial layer surrounding the individual nerve fascicles [14].

Imaging characteristics in this case were indeterminate, leading to a fairly broad differential diagnosis. Benign peripheral nerve sheath tumors (schwannoma and neurofibroma) have a similar clinical presentation, often showing significant hyperintensity on fluid-weighted sequences and are less elongated. They also may have a central area of low signal intensity, or "target sign," which was not present $[1,20]$. Owing to the elongated fusiform nature, focal chronic inflammatory demyelinating polyneuropathy was a consideration. Chronic inflammatory demyelinating polyneuropathy may present as a local progressive neuropathy without denervation changes, is isointense on T1weighted sequences, hyperintense on T2-weighted sequences, and can show subtle peripheral enhancement [1, 20]. Schwannomas, neurofibromas, and chronic inflammatory demyelinating polyneuropathy all histologically 
stain negative for epithelial membrane antigen and are S100 positive [5]; the reverse was seen in our case.

The nonspecific characteristics also raised the suspicion of lymphoma involving the peripheral nerve. Lymphoma can present as a mononeuropathy $[7,8]$ with no other apparent disease [20]. On MRI the nerve often appears mildly enlarged with an irregular to nodular appearance, mild to moderate hyperintensity on T2-weighted sequences, and mild enhancement of the entire nerve [20]. The diagnosis was excluded when hematoxylin and eosin-stained biopsy sections did not show the large, pleomorphic, and mitotically active lymphoid cells seen with lymphoma [7, 8].

Malignant peripheral nerve sheath tumors are rare and ordinarily seen with syndromes (neurofibromatosis) or after radiation therapy [20]. They are difficult to diagnose owing to nonspecific imaging characteristics, histologic variation, and nonstandardized criteria for diagnosis $[15,20]$. Irregular margins, nonenhancing areas not related to the "target sign" or speckling seen in benign lesions, and atrophy in muscles should increase suspicion for possible malignancy [20]. The lack of central enhancement seen on MR images was concerning for a possible malignant peripheral nerve sheath tumor; however, this was excluded based on our histology results. Characteristic histologic features of malignant peripheral nerve sheath tumors include wavy, buckled, or comma-shaped nuclei, a fascicular spindle cell proliferation with an abrupt transition between cellular and myxoid areas, perivascular accentuation (whirling of tumor cells around a vessel), and heterologous differentiation may be seen in $10 \%$ to $15 \%$ of cases (including rhabdomyosarcoma, osteosarcoma, chondrosarcoma, angiosarcoma, and or glandular differentiation), which were not present in our patient $[9,15]$.

Intraneural perineurioma is a benign lesion of neoplastic perineurial cells, and to our knowledge, there have been no reports of this tumor undergoing malignant transformation. However, an intraneural perineurioma often is progressive, leading to weakness and potentially permanent functional impairment $[5,12,15]$. Surgical extirpation, with marginal or conservative wide excision, is definitive with no reported recurrence $[5,13]$, but because resection requires removal of all or part of the nerve, postoperative impairment is expected. Gruen et al. [12] recommended surgical resection and autologous nerve grafting if no action potentials or low amplitude recordings were noted intraoperatively. In the literature review by Boyanton et al. [5], they reported on 16 patients treated with surgical resection and nerve grafting or primary anastomosis; $31 \%$ (five) reported no improvement and 69\% (11) showed some improvement in motor function. In 2009, Mauermann et al. [13] reported on 13 patients who underwent surgical interventions (eight tendon transfers without lesion resection; three tendon transfers with lesion resection and nerve grafting; and two lesion resections and nerve grafting without tendon transfer) with followup information for seven patients. Four patients experienced improvement in strength and function, two experienced initial improvement followed by progression of weakness, and one who had postoperative arthrofibrosis develop despite further treatment never showed improvement. Mauermann et al. [13] also noted that 12 patients had slow progression of sensory and motor deficits during a 3.5-year period. They believed the patients' tumors were slowly progressing if not static, having a low morbidity, and probably should not be resected routinely.

Cortes et al. [6], based on a 9-year-old patient with good results postoperatively, recommended early surgical resection with reconstruction in the young patient. Improved recovery of function in children and adolescents compared with adults, regardless of method of repair, duration, or location of injury, has been reported [3, 6, 21-24]. However, contradicting evidence also has shown disappointing outcomes in adolescents who were treated with surgical resection $[6,18,19]$.

Our patient was referred to a peripheral nerve surgeon for consultation regarding surgical resection. It has been 1 year since her diagnosis was made and she has yet to pursue further intervention.

Acknowledgments We thank Sarah George MD (Department of Pathology, McLaren Macomb Medical Center) for input with the histology interpretation and Tracy Chen DO (Department of Radiology, McLaren Macomb Medical Center) for input with the radiology interpretation.>

\section{References}

1. Amrami KK, Felmlee JP, Spinner RJ. MRI of peripheral nerves. Neurosurg Clin N Am. 2008;19:559-572.

2. Ariza A, Bilbao JM, Rosai J. Immunohistochemical detection of epithelial membrance antigen in normal perineurial cells and perineurioma. Am J Surg Pathol. 1988;12:678-683.

3. Barfred T, Zachariae L, Neurofibroma in the median nerve treated with resection and free nerve transplantation: case reports. Scand J Plast Reconstr Surg. 1975;9:245-248.

4. Beekman R, Slooff WB, Van Oosterhout MF, Lammens M, Van Den Berg LH. Bilateral intraneural perineurioma presenting as ulnar neuropathy at the elbow. Muscle Nerve. 2004;30:239-243.

5. Boyanton BL Jr, Jones JK, Shenaq SM, Hicks MJ, Bhattacharjee MB. Intraneural perineurioma: a systematic review with illustrative cases. Arch Pathol Lab Med. 2007;131:1382-1392.

6. Cortes W, Cheng J, Matloub HG. Intraneural perineurioma of the radial nerve in a child. $J$ Hand Surg Am. 2005;30:820-825.

7. Del Grande A, Sabatelli M, Luigetti M, Conte A, Granata G, Rufini V, Del Ciello A, Gaudino S, Fernandez E, Hohaus S, Coli A, Lauriola L. Primary multifocal lymphoma of peripheral nervous system: case report and review of the literature. Muscle Nerve. 2014;50:1016-1022.

8. Descamps MJ, Barrett L, Groves M, Yung L, Birch R, Murray NM, Linch DC, Lunn MP, Reilly MM. Primary sciatic nerve lymphoma: a case report and review of the literature. J Neurol Neurosurg Psychiatry. 2006;77:1087-1089. 
9. Doyle LA, Nascimento AF. Soft tissue tumors: malignant peripheral nerve sheath tumors. In: Cheng L, Bostwick DG, eds. Essentials of Anatomic Pathology. 3rd ed. New York, NY: Springer; 2011:995-1045.

10. Emory TS, Scheithauer BW, Hirose T, Wood M, Onofrio BM, Jenkins RB. Intraneural perineurioma: a clonal neoplasm associated with abnormalities of chromosome 22. Am J Clin Pathol. 1995;103:696-704.

11. Groeneweg AJ, Hartman EH, Fleischneuer R, Visser LH. An unusual location of ulna nerve pathology: a perineurioma of the ulnar nerve in the upper arm. Muscle Nerve. 2011;44:593-596.

12. Gruen JP, Mitchell W, Kline DG. Resection and graft repair for localized hypertrophic neuropathy. Neurosurgery. 1998;43: 78-83.

13. Mauermann ML, Amrami KK, Kuntz NL, Spinner RJ, Dyck PJ, Bosch EP, Engelstad J, Felmlee JP, Dyck PJ. Longitudinal study of intraneural perineurioma: a benign, focal hypertrophic neuropathy of youth. Brain. 2009;132:2265-2276.

14. Nacey NC, Suarez MIA, Mandell JW, Anderson MW, Gaskin CM. Intraneural perineurioma of the sciatic nerve: an underrecognized nerve neoplasm with characteristic MRI findings. Skeletal Radiol. 2014;43:375-379.

15. Rosenberg AS, Langee CL, Stevens GL, Morgan MB. Malignant peripheral nerve sheath tumor with perineurial differentiation: 'malignant perineurioma'. J Cutan Pathol. 2002;29:362-367.
16. Sachanandani NS, Brown JM, Zaidman C, Brown SS, Mackinnon SE. Intraneural perineurioma of the median nerve: case report and literature review. Hand (NY). 2010;5:286-293.

17. Scheller C, Richter HP, Scheuerle A, Kretschmer T, Konig RW, Antoniadis G. Intraneural perineuriomas; a rare entity: clinical, surgical, and neuropathological details on the management of these lesions. Zentralbl Neurochir. 2008;69:134-138.

18. Simpson DA, Fowler M. Two cases of localized hypertrophic neurofibrosis. J Neurol Neurosurg Psychiatry. 1966;29:80-84.

19. Snyder M, Cancilla PA, Batzdorf U. Hypertrophic neuropathy simulating a neoplasm of the brachial plexus. Surg Neurol. 1977;7:131-134.

20. Spinner RJ, Amrami KK. What's new in the management of benign peripheral nerve lesions? Neurosurg Clin $N$ Am. 2008;19:517-531.

21. Stevenson JH, Zuker RM. Upper limb motor and sensory recovery after multiple proximal nerve injury in children: a long term review in five patients. Br J Plast Surg. 1986;39:109-113.

22. Tajima $\mathrm{T}$, Imai H. Results of median nerve repair in children. Microsurgery. 1989;10:145-146.

23. Tyagi A, Drake J, Midha R, Kestle J. Axillary nerve injuries in children. Pediatr Neurosurg. 2000;32:226-229.

24. Vahvanen V, Gripenberg L, Nuutinen P. Peripheral nerve injuries of the hand in children: a follow-up study of 38 patients. Scand $J$ Plast Reconstr Surg. 1981;15:49-51. 